\title{
Study the Corpus Callosum of Brain to Explore Autism Employing Image Segmentation
}

\author{
Mousumi Bala ${ }^{1, *}$, Suraiya Yasmin ${ }^{2}$ \\ ${ }^{1}$ Central Women's University, Dhaka, Bangladesh \\ ${ }^{2}$ Bangabandhu Sheikh Mujibur Rahman Agricultural University, Bangladesh
}

Copyright $(2016$ by authors, all rights reserved. Authors agree that this article remains permanently open access under the terms of the Creative Commons Attribution License 4.0 International License

\begin{abstract}
Autism is a lifetime developmental disability which is a great concern in the present world. As of 2010, autism affects 1-2 in every 1000 people all over the world. Autism is highly inherited that appears during infancy or childhood. It is a spectrum condition; some people have learning disabilities, mental health issues and other conditions like hearing impairment, Down's syndrome, epilepsy etc. Recent studies show autistic people encompass faulty connections between different brain regions; corpus callosum region is one of them. To explore the causes of autism, the study of corpus callosum of brain is carried out among the control and autistic individuals in which fMRI (functional Magnetic Resonance Imaging) scan images of corpus callosum is taken as input. Firstly, Sobel, Prewitt and Robert edge detection is performed in the images respectively to detect the surrounding edges of corpus callosum and then thresholding method is applied for segmentation of images. The work is carried out for the children, teenager and adult. Furthermore, the binarization method is used to calculate the area of corpus callosum of brain for the control and autistic patients. Finally, the differences of the produced images of corpus callosum between the control and autistic individuals will help in understanding autism.
\end{abstract}

Keywords ASD (Autism Spectrum Disorder), Autism, Corpus Callosum, Hemisphere, fMRI (Functional Magnetic Resonance Imaging)

\section{Introduction}

Autism is an invasive developmental disorder which includes abnormal development and function of the brain. Autistic people have decreased social communication skills and restricted or repetitive behavior or interest. The causes of autism remain unknown, many studies have concerned a variety of developmental genes, environmental factors such as exposures to viruses or toxins during pregnancy are hypothesized to contribute but none have been decisively identified [1]. Children with autistic disorder have complexities relating to and communicating with other people. When they're babies, they do not look at other people. They do not respond to their name or smile at others at the age of two years [2]. They might not change their pitch when they're babbling, so it doesn't sound like a conversation. They do not impersonate others with behavior also like clapping or waving. Older children and teenagers with autism spectrum disorder (ASD) typically have trouble with both verbal and non-verbal communication for social purposes. An older child or teenager with ASD might have difficulty taking turns in conversations - for example, she might like to do all the talking or find it hard to answer the questions. They might talk a lot about a favorite topic, but find it difficult to talk about a variety of topics. They also have an abnormal tone of voice, or use speech in an unusual way - for example, she might speak in a monotone or with an accent. They make use of unusual eye contact, express few emotions in their face- for example they might not be able to tell if someone likes them in a romantic way and also they employ very few gestures to express themselves [3].

To comprehend autism and their remedies, a number of research papers [4]-[8] have been published. Many researchers studied autism [9]-[12] in view of corpus callosum of the brain as major culprit.

The functional magnetic resonance imaging (fMRI) is a functional neuroimaging procedure using MRI technology that is mainly used for the delineation of changes of local blood supply in the brain activated by specific stimuli [13]. The primary form of fMRI uses the blood-oxygen-level dependent (BOLD) contrast, discovered by Seiji Ogawa. This is a sort of specialized brain and body scan which is used to map neural activity in the brain or spinal cord of humans or other animals by imaging the change in blood flow (hemodynamic response) related to energy utilize by brain cells [14].

The Sobel, Robert and Prewitt operator is used to detect edges based applying a horizontal and vertical filter in sequence. Both filters are applied to the image and summed to form the final result. The Roberts Edge detector is fast 
since the filter is small but it is also issue to interference by noise .If edges are not very sharp the Robert filter will tend not to detect the edge [15].The Sobel operator, sometimes called the Sobel-Feldman operator or Sobel filter, is used in image processing and mostly within edge detection algorithms where it creates an image emphasizing edges. Theoretically, it is a discrete differentiation operator, computing an approximation of the gradient of the image intensity function [16]. The Sobel operator is a discrete operator, which computes the gradient for intensity changes at each point in an image. The operator consists of a pair of $3 * 3$ convolution kernels. The Roberts operator performs a simple, quick to compute, two-dimensional (2-D) spatial gradient measurement on an image. The operator consists of a pair of $2 * 2$ convolution kernels [17]. Prewitt operator is a discrete differentiation operator which functions similar to the Sobel operator, by computing the gradient for the image intensity function.

The Sobel, Prewitt and Robert operators are used to detect the edges of brain surrounding corpus callosum for both control and autistic patients. To carry out these works the fMRI scan images of children, teenager and adult are taken as input. Using Sobel operator for extraction corpus callosum area of brain both control and autistic using segmentation methods. Because segmentation process attempts to partition an image into its constituent parts or objects. The purpose of segmentation is to isolate the objects or parts of interest from an image [18].

\subsection{Corpus Callosum}

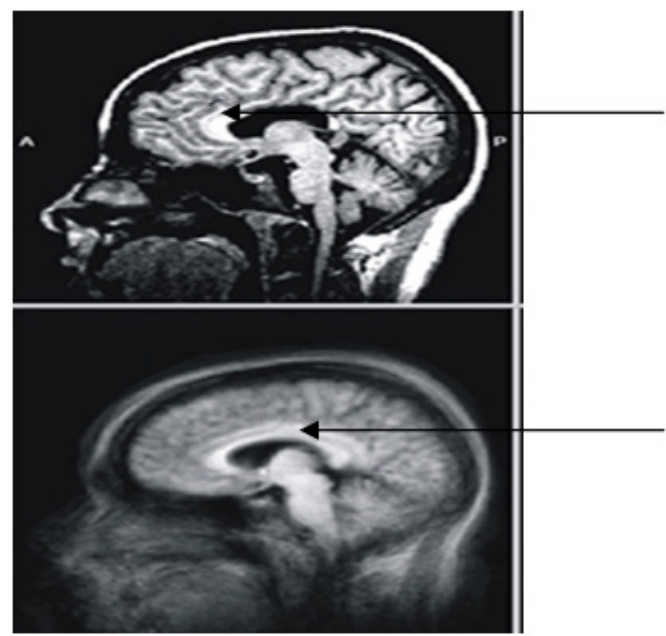

Autism

Control

Figure 1. Corpus callosum of brain for control and autistic.

Corpus callosum consists of about 200 million axons that interconnect the two hemispheres of brain [19]. Each hemisphere of the brain is specialized to control movement and feeling in the opposite half of the body, and each hemisphere specializes in processing certain types of information (such as language or spatial patterns). To coordinate movement or to think about complex information, the hemispheres must communicate with each other. The corpus callosum acts like a bridge that allows that communication. Disorders of the corpus callosum are not illnesses or diseases, but the brain abnormalities of human [20]. Many people with these conditions are healthy. However, other individuals with disorders of the corpus callosum do require medical intervention due to seizure and/or other medical problems they have in addition to the corpus callosum disorder. Figure1 shows the corpus callosum of brain for the both control and autistic which clearly indicates the differences among them (the shape of autistic is not very lucid).

\section{Materials and Methods}

In carrying out the work fMRI scan images of the corpus callosum of brain for control and autistic individuals are taken as input. The work is carried out for the children, teenager and adult for both control and autistic individuals. Roberts, Prewitt and Sobel operators are used for the edge detection of brain images.

\section{Roberts Operator}

The Roberts operator is given by the equations:

$\mathrm{G}_{\mathrm{x}}=\mathrm{W}_{9}-\mathrm{W}_{5}$

$$
\mathrm{G}_{\mathrm{y}}=\mathrm{W}_{8}-\mathrm{W}_{6}
$$

\begin{tabular}{|c|c|}
\hline-1 & 0 \\
\hline 0 & 1 \\
\hline
\end{tabular}

(a)

\begin{tabular}{|c|c|}
\hline 0 & -1 \\
\hline 1 & 0 \\
\hline
\end{tabular}

(b)

Figure 2. (a) Roberts mask for horizontal direction(b) Roberts mask for vertical direction

\section{Sobel Operator}

Consider the arrangement of pixels about the pixel:

\begin{tabular}{|c|c|c|}
\hline $\mathrm{W}_{1}$ & $\mathrm{~W}_{2}$ & $\mathrm{~W}_{3}$ \\
\hline $\mathrm{W}_{4}$ & $\mathrm{~W}_{5}$ & $\mathrm{~W}_{6}$ \\
\hline $\mathrm{W}_{7}$ & $\mathrm{~W}_{8}$ & $\mathrm{~W}_{9}$ \\
\hline
\end{tabular}

The Sobel operator is given by the equations:

$\mathrm{G}_{\mathrm{x}}=\left(\mathrm{W}_{7}+2 \mathrm{~W}_{8}+\mathrm{W}_{9}\right)-\left(\mathrm{W}_{1}+2 \mathrm{~W}_{2}+\mathrm{W}_{3}\right)$

$\mathrm{G}_{\mathrm{Y}}=\left(\mathrm{W}_{3}+2 \mathrm{~W}_{6}+\mathrm{W}_{9}\right)-\left(\mathrm{W}_{1}+2 \mathrm{~W}_{4}+\mathrm{W}_{7}\right)$

Where, $\mathrm{W}_{1}$ to $\mathrm{W}_{9}$ are pixels values in a sub image as shown in Figure 3

\begin{tabular}{|c|c|c|}
\hline-1 & -2 & -1 \\
\hline 0 & 0 & 0 \\
\hline 1 & 2 & 1 \\
\hline
\end{tabular}

(a)

\begin{tabular}{|c|c|c|}
\hline-1 & 0 & 1 \\
\hline-2 & 0 & 2 \\
\hline-1 & 0 & 1 \\
\hline
\end{tabular}

(b)

Figure 3. (a) Sobel mask for horizontal direction; (b) Sobel mask for vertical direction 


\section{Prewitt Operator}

The Prewitt's operator is given by the equations:

$\mathrm{G}_{\mathrm{x}}=\left(\mathrm{W}_{7}+\mathrm{W}_{8}+\mathrm{W}_{9}\right)-\left(\mathrm{W}_{1}+\mathrm{W}_{2}+\mathrm{W}_{3}\right)$

$\mathrm{G}_{\mathrm{Y}}=\left(\mathrm{W}_{3}+\mathrm{W}_{6}+\mathrm{W}_{9}\right)-\left(\mathrm{W}_{1}+\mathrm{W}_{4}+\mathrm{W}_{7}\right)$

\begin{tabular}{|c|c|c|}
\hline-1 & -1 & -1 \\
\hline 0 & 0 & 0 \\
\hline 1 & 1 & 1 \\
\hline
\end{tabular}

(a)

\begin{tabular}{|c|l|l|}
\hline-1 & 0 & 1 \\
\hline-1 & 0 & 1 \\
\hline-1 & 0 & 1 \\
\hline
\end{tabular}

(b)

Figure 4. (a) Prewitt mask for horizontal direction (b) Prewitt mask for vertical direction

Extraction of the activation area of corpus callosum is done using thresholding. Then these images are taken as input for Segmentation. After segmentation, the values of activation areas of both control and autistic individuals are calculated using the binarization method. That is the image having only two values either black or white ( 0 or 1$)$. Here $256 \times 256$ jpeg image is a maximum image size. The binary image can be represented as a summation of total number of white and black pixels. Area of an image is the total number of the pixels present in the area which can be calculated in the length units by multiplying the number of pixels with the dimension of one pixel:

$$
\begin{aligned}
& \text { Image, } \mathrm{I}=\Sigma 255 w=0 \Sigma 255 H=0[\mathrm{f}(0)+\mathrm{f}(1)] \\
& \mathrm{f}(0)=\text { white pixel (digit } 0) \\
& \mathrm{f}(\mathrm{l})=\text { black pixel (digit } 1) \\
& \text { Pixels = Width (W) X Height }(\mathrm{H}) \\
& =256 \text { X } 256
\end{aligned}
$$

No_of_white pixel $\mathrm{P}=\Sigma 255 w=0 \Sigma 255 H=0[\mathrm{f}(0)]$ Where, $\mathrm{P}=$ number of white pixels (width*height)

Finally, the calculation of activation values is used for comparing the surroundings of corpus callosum to show the graphical representation for both control and autistic patients. The proposed work is implemented using Matlab.

\section{Result and Discussion}

The work is performed to explore the biological aspects of autism. In carrying out the work fMRI scan images of children, teenager and adult are taken as input and the corresponding images are produced using edge detection methods (Roberts, Prewitt and Sobel), thresholding, segmentation and binarization operation respectively. The purpose of applying these image processing methods is to calculate the activation areas of corpus callosum. The images of the input and produced output for both control and the autistic individuals are shown separately. Figure 5 shows the input images of the corpus callosum of brain both of control and autistic for children, teenager and adult.

\section{Roberts Operator}

To detect the neural pathway surrounding the corpus callosum area of brain, Roberts operator is used for the control and the autistic in. Figure 6 shows the processed images of the corpus callosum of brain for control and autistic patients. The Roberts operator is used on input images and the output images of corpus callosum of children, teenager and adult are produced. Comparing the produced images, it is clearly visualized that the edges for the control are sharply observed which indicates that the neural pathways for the control are more effective surrounding the corpus callosum than autistic.

\section{Prewitt Operator}

Prewitt operator is used also for both control and the autistic individuals to detect the neural pathways of brain. Figure 7 shows the processed images of corpus callosum of brain for children, teenager and adult. Comparing the produced images, it is clearly visualized that the edges for the control are sharply observed where the edges surrounding corpus callosum of brain are not very sharp in autistic. The neural pathways are less effective in autistic brain than control.

\section{Sobel Operator}

The main difference between Robert and Sobel is the kernels. The Sobel kernels are more suitable to detect edges horizontally and vertically rather than Robert's [21]. However, the Prewitt operators have a major drawback of being sensitivity of noise. Sobel edge detection operator is applied on the input images of corpus callosum of brain for the control and autistic patients and the corresponding images are produced which is shown in Figure 8. The produced images are sharply observed by using Sobel edge detection operator rather than Robert and Prewitt. Comparing the produced images of children, teenager and adult shown in Figure 8, the neural pathways for the control are clearly visualized and it indicates that neurons are more effective in control than autistic.

\section{Thresholding}

Figure 9.shows the result of thresholding which gives the accurate edge detected images from processed images using Sobel operator of the corpus callosum of brain for control and autistic for children, teenager and adult.

\section{Segmentation}

It has been observed that Sobel edge detection operator is computationally more expensive compared to Prewitt and Robert's operator. It means using Sobel operator the edge detection of corpus callosum of brain is very clear from Roberts and Prewitt operators. Consider processed images using Sobel operator for extraction corpus callosum area of brain both control and autistic using segmentation methods. Figure 10.shows the segmented images for both control and autistic individuals. It also shows extracted activation area of corpus callosum of brain for both control and autistic individual. 
Children

Control

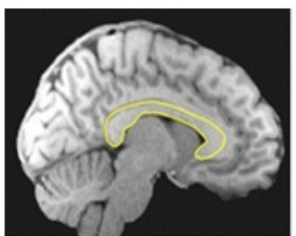

Autism

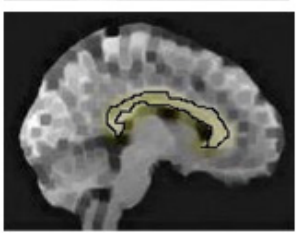

Teenager
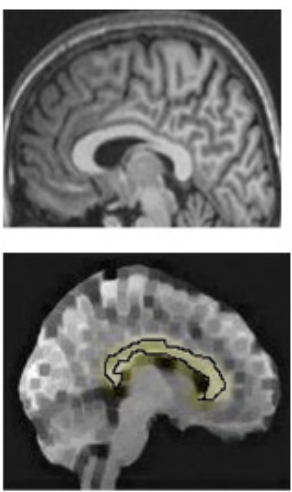

Adult
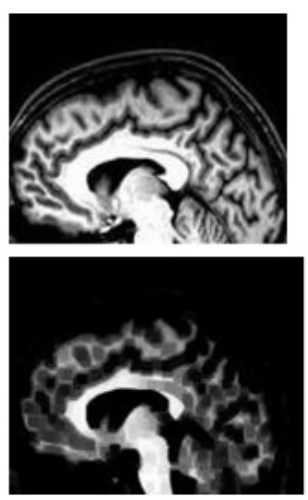

Figure 5. fMRI images of corpus callosum of control and autistic for children, teenager and adult.

Children

Control

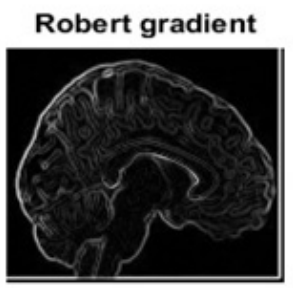

Autism

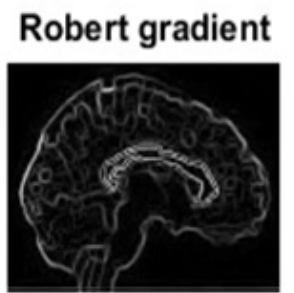

\section{Teenager}

Robert gradient

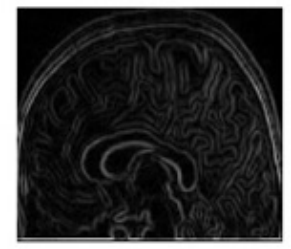

Robert gradient

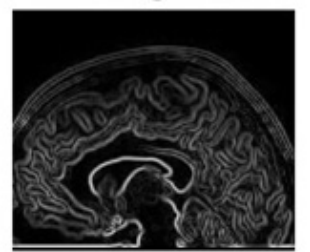

Adult

Robert gradient

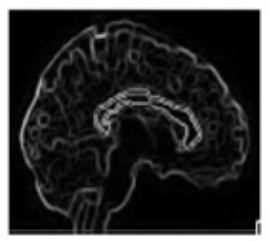

Robert gradient

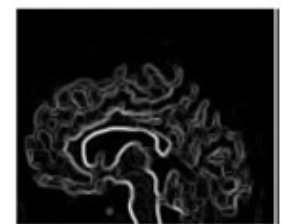

Figure 6. Processed images of the corpus callosum using Roberts operator of control and autistic for children, teenager and adult.

Children

Control

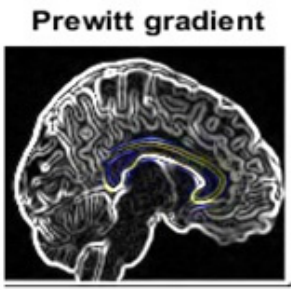

Autism
Teenager

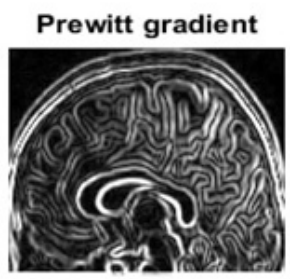

Prewitt gradient

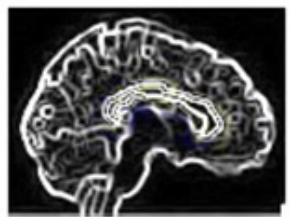

Adult

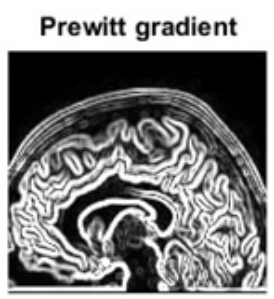

Prewitt gradient

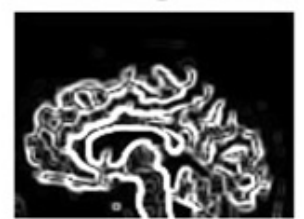

Figure 7. Processed images of the corpus callosum using Prewitt operator of control and autistic for children, teenager and adult. 
Children

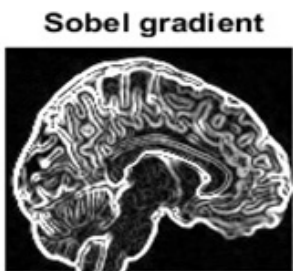

Sobel gradient

Autism

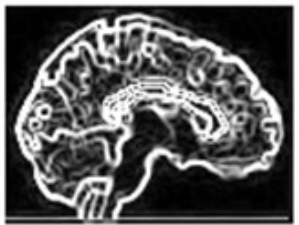

Teenager

Sobel gradient

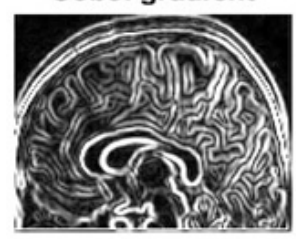

Sobel gradient

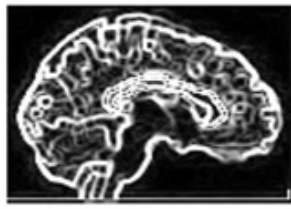

Adult

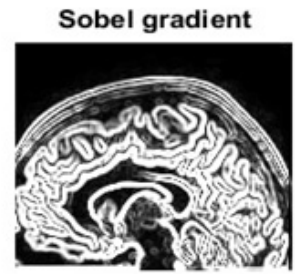

Sobel gradient

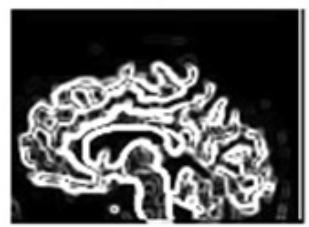

Figure 8. Processed images of the corpus callosum using Sobel operator of control and autistic for children, teenager and adult.

Children

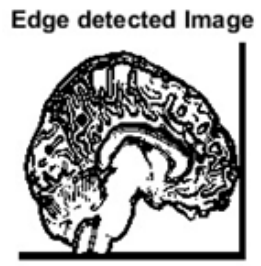

Edge detected Image

Autism

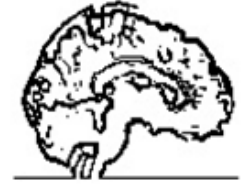

Teenager

Edge detected Image

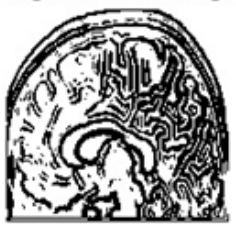

Edge detected Image

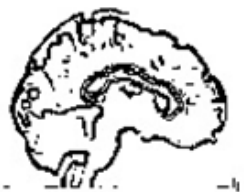

Adult

Edge detected Image

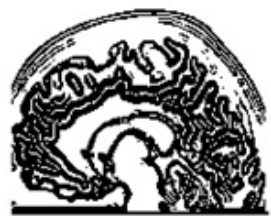

Edge detected Image

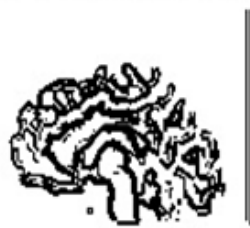

Figure 9. Edge detected images of the corpus callosum of brain for control and autistic using thresholding.

Children

Control

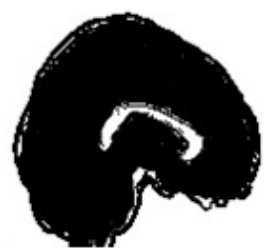

Autism

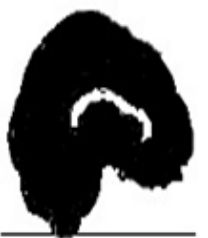

Teenager
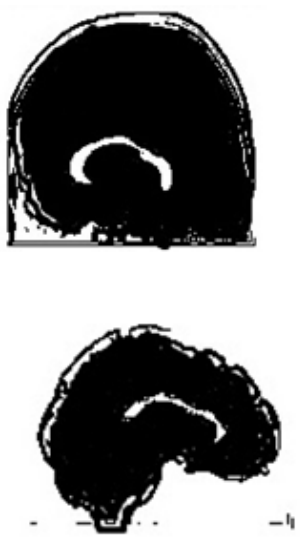

Adult
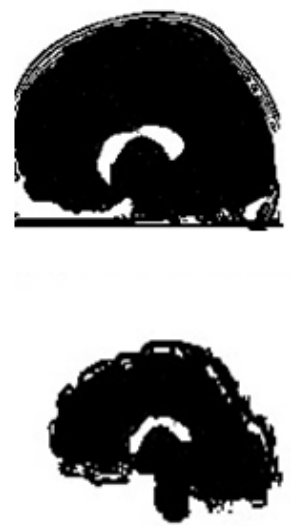

Figure 10. Segmented images] of the corpus callosum of brain of both control and autistic for children, teenager and adult. 
Table 1. Indicated activat]ion area and calculated values of both control and autism for children.

\begin{tabular}{|c|c|c|}
\hline \multirow{2}{*}{ For children } & Control & Autistic \\
\hline & & 396
\end{tabular}

Table 2. Indicated activation area and calculated values of both control and autism for teenager.

For teenager

Table 3. Indicated activation area and calculated values of both control and autism for adult.

\begin{tabular}{|c|c|c|}
\hline \multirow{2}{*}{ For adult } & Control & Autistic \\
\hline Area in pixels & & \\
\hline
\end{tabular}

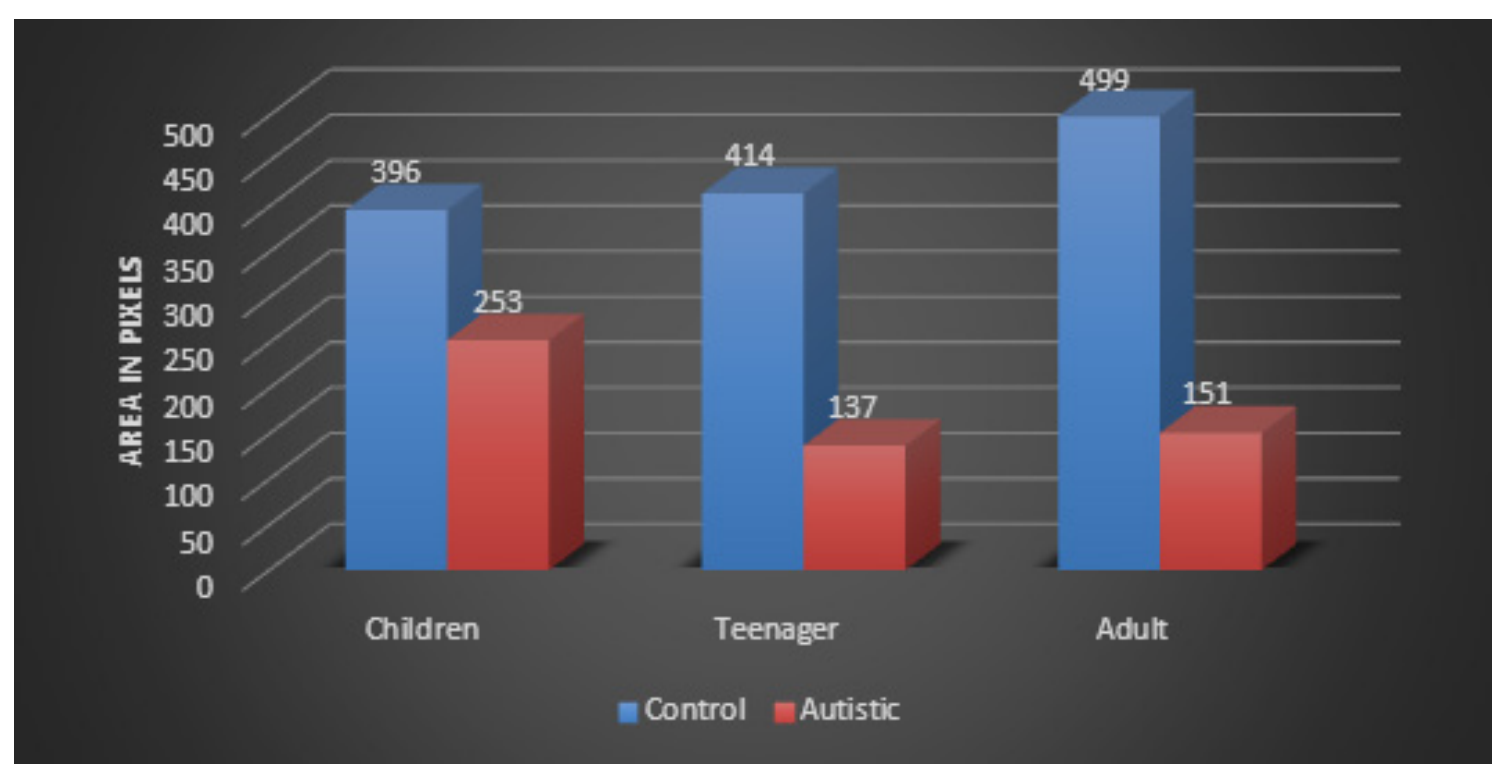

Figure 11. Graphical representation of both control and autistic for children, teenager and adult. 


\section{Calculate the Area of Corpus Callosum}

Consider segmented processed images for calculating the values using binarization operation of activation area corpus callosum of brain of control and autistic for children, teenager and adult.

Table 1.shows activation area is indicated as pink color of corpus callosum of brain both of control and autistic for children. The values are calculated for indicated area of corpus callosum. The calculated values is 396 (in pixels) for control and 253 (in pixels) for autistic. From these calculated values, it is observed that the values of corpus callosum of autistic are reduced than control.

Table 2.also shows activation area is indicated as pink color of corpus callosum of brain both of control and autistic for teenager. The values are calculated for indicated area of corpus callosum. The calculated values are 414 (in pixels) for control and 137 (in pixels) for autistic. From these calculated values, it is observed that the values of corpus callosum of autistic are reduced than control.

Table 3.also shows activation area is indicated as pink color of corpus callosum of brain both of control and autistic for adult. The values are calculated for indicated area of corpus callosum. The calculated values are 499 (in pixels) for control and 151 (in pixels) for autistic. It is observed that the values of corpus callosum of autistic are reduced than control.

From the calculating values, it has been observed that the values of activation area for autistic are smaller than control. The compared results are shown in Figure 11 with graphical representations.

\section{Conclusions}

By using Sobel operator the edge detection of corpus callosum of brain is very clear from Roberts and Prewitt operators. Then applying threshoding, segmentation and binarization operation on fMRI images of control and autistic individuals, it is clearly visualized that there are differences in calculated values of activation area of the corpus callosum for children, teenager and adult. From these differences, it has been observed that the activation areas in corpus callosum are hypoactive in patient with autism than in control. As the activation area extracted from the corpus callosum and its segments is significant, the results of this study could be used to assess the detection human neurological disorders such as autism. It is a simulation model to understand the risk of autistic individuals. To understand more about autism, functional studies of corpus callosum and its surroundings are of key interest and molecular level investigations are needed for further detailed study.

\section{REFERENCES}

[1] “Autism Information", [online] Available: https://autism-cen ter.ucsd.edu/autism-information/Pages/what-is-autism.aspx, Accessed: [November 28, 2016].

[2] “Autistic disorder", [online] Available: http://raisingchildren .net.au/articles/autistic_disorder_signs_and_symptoms.html, Accessed: [November 29, 2016]

[3] "Sign of autism spectrum disorder in older children and teenagers", [Online] Available: http://raisingchildren.net.au/ articles/autistic_disorder_signs and symptoms.html, Accessed: [November 29, 2016]

[4] American Psychiatric Association, "Diagnostic and Statistica 1 Manual of Mental Disorders" (4th ed.) Washington, DC: American Psychiatric Association, 2000.

[5] Carlson, N. R, "Physiology of Behavior" (9th ed.). United States of America: Pearson Education, Inc, 2007.

[6] Castelli, F., Frith, C., Happé, F., Frith, U, “Autism, Asperger syndrome and brain mechanisms for the attribution of mental states to animated shapes", Oxford University Press, 125(8), 1839-1849,2002, doi: 10.1093/brain/awf189

[7] Green, G., Taylor, B., Luce, S., Krantz, P, “Applied behavior analysis" Retrieved from www.autismspeaks.org/what-autis $\mathrm{m} /$ treatment/applied-behavioral-analysis-aba, 2012.

[8] M. A. Just, V. L. Cherkassky, T. A. Keller, N. J. Minshew, "Cortical activation and synchronization during sentence comprehension in high-functioning autism: evidence of underconnectivity”, Brain vol. 127, pp. 1811-1821, 2004.

[9] SuraiyaYasmin and Mohammad Hanif Ali "Analysis of Corpus Callosum of Brain to Explore Autism" Vol. EE 40, No. I \& II, June \& December, 2014

[10] Q. He, Ye Duan, K. Karsch and J. Miles. "Detecting corpus callosum abnormalities in autism based on anatomical landmarks", vol. 183, no. 2, pp. 126-132, 2010.

[11] J. Piven, J. Bailey, Bonny, J. Ranson, and Stephen Arndt, “An MRI Study of the Corpus Callosum in Autism" vol. 154, no. 8, pp. 1051-1056, 1997.

[12] Mara Fabri, Chiara Pierpaoli, Paolo Barbaresi, and Gabriele Polonara, "Functional Topography of the Corpus Callosum Investigated by DTI and fMRI", vol. 6(12): 895-906, 2014.

[13] "Magnetic Resonance, a critical peer-reviewed introduction; functional MRI". European Magnetic Resonance Forum, [online], Available:http://www.magnetic-resonance.org/ch/1 1-03.html, Accessed: [November 29, 2016].

[14] Huettel, S. A.; Song, A. W.; McCarthy, G. "Functional Magnetic Resonance Imaging"(2 ed.), Massachusetts: Sinauer,ISBN978-0-87893-286-3, 2009.

[15] "Roberts Edge", [Online], Available: http://www.roborealm. com/help/Roberts_Edge.php, Accessed: [November 29, 2016]

[16] Irwin Sobel, History and Definition of the Sobel Operator, 2014.

[17] Mehravar Rafati, Masoud Arabfard, and Mehrdad Rafati-Rahimzadeh, "Comparison of Different Edge Detections and Noise Reduction on Ultrasound Images of Carotid and Brachial Arteries Using a Speckle Reducing Anisotropic Diffusion Filter", vol. 16(9), 2014, doi: 10.5812/ircmj. 14658 
[18] Rafael C. Gonzalez. "Digital Image Processing using MATLAB".

[19] “Corpus Callosum” [Online], Available: http://cnsvp.stanfor d.edu/atlas/corpus_callosum.html, Accessed: [November 29, 2016].
[20] "Corpus Callosum Disorder", National organization for disorders of the corpus callosum, [Online], Available: http://nodcc.org/corpus-callosum-disorders, Accessed: [Nov ember 29, 2016].

[21] S. Dewangan and P. K. Gupta. "Evaluation of edge detection techniques on photographic images, vol. 3, no. 7, 2012. 